\title{
MANUAL DIDÁCTICO EN SOPORTE VIRTUAL PARA LA PRÁCTICA DE LA TRADUCCIÓN ESPECIALIZADA
}

\section{DIDACTIC HANDBOOK IN VISUAL FORMAT FOT THE PRACTICE OF SPECIALIZED TRANSLATION}

\author{
Soledad Díaz Alarcón, Carmen Arnedo Villaescusa, Azahara Veroz González, Ángeles García Calderón,
} Beatriz Martínez Ojeda, Rafael Porlán Moreno, Mª del Mar Ogea Pozo, Lourdes Bonhome,

Fuensanta Guerrero Carmona

1r2dials@uco.es

Universidad de Córdoba

Received: 20/07/2017

Accepted: 04/12/2017

\section{Abstract}

The teaching experience of the members of this project emphasises the importance of directing teaching principles at encouraging students' motivation and commitment within the learning process, as well as specifying and classifying methodologies and materials in order to adapt them to the necessities and capabilities of the translation students. This fact gives rise to the initiative to provide students a fully-designed, progressive and practical methodology, which is based on the models for discourse analysis and translation analysis.

In this regard, the present project targets the elaboration of teaching materials for the practice of specialised translation. In particular, we have designed a virtual manual whose contents are presented through explanatory videos. These audiovisual contents, together with texts, bibliographical resources, analysis models and commented translations, have been hosted in the EXA ENOA2 virtual platform - with open-access to all students at the University of Córdoba - in html5 format, so it can be accessed from any mobile device.

The manual includes four itineraries (legal-economical, scientific-technical, humanistic-literary, and audiovisual). It offers a specific discourse analysis model for each field, helping the student to detect and prioritize the characteristics of each textual genre and their linguistic features.

These analysis models are applied to a bilingual corpora (FR/EN) of texts selected and explained by the teachers. Moreover, a Spanish translation and the corresponding translation analysis are provided.

The manual also procures an annex including a selection of documentary resources in electronic format - such as dictionaries, termbases, specialized translation guides, etc. - that students can check immediately or download for future consults.

This open-access virtual material aims to be a self-learning tool for specialised translation and to be constituted as a teaching complement for further acquisition of translation skills in specialised fields, as well as a practical and functional methodology that enables students to optimise every phase of the translation process.

Keywords: Virtual manual; Specialised translation; Translation techniques.

\section{Resumen}

La experiencia docente de los participantes en este proyecto ha puesto de relieve la importancia de orientar sus principios didácticos a fomentar la incentivación y el compromiso de los alumnos en el proceso de aprendizaje, así como la explicitación y ordenación de metodologías y materiales, adecuándolos a las necesidades y capacidades del alumno de traducción. De ahí, surge la iniciativa de proporcionar al alumno una metodología práctica bien definida, de carácter progresivo, y sustentada en modelos de análisis de discurso y de análisis traductológico.

Según esto, este proyecto se orienta a la creación de material docente para la práctica de la traducción especializada. En concreto se ha diseñado un manual virtual cuyo contenido se presenta mediante vídeos explicativos. Este material audiovisual se ha alojado, junto a los textos, recursos bibliográficos, modelos de análisis, traducciones comentadas, etc., en la plataforma virtual EXA ENOA2, de acceso abierto a los alumnos de la Universidad de Córdoba y con formato html5, para poder acceder a ella desde cualquier dispositivo móvil.

El manual, estructurado en cuatro itinerarios (jurídico-económico-administrativo, científico-técnico, humanístico-literario y audiovisual), ofrece un modelo de análisis discursivo específico que permite al alumno detectar y priorizar las especificidades de cada género textual y sus particularidades lingüísticas. Estos análisis se aplican a un corpus bilingüe de textos (FR $>$ ES / EN $>$ ES) seleccionados y explicados por los docentes, de los que se aporta además una traducción al español y su correspondiente análisis traductológico.

Este manual proporciona además, a modo de anexo, una selección de recursos documentales, en formato electrónico, de carácter especializado (diccionarios, bases terminológicas, manuales de traducción especializada, etc.) que el alumno puede consultar de modo inmediato o descargarse en su ordenador para futuras consultas.

Este material en soporte virtual de acceso abierto pretende configurarse como manual de autoaprendizaje de técnicas de traducción especializada y se constituye como complemento didáctico para el perfeccionamiento de las destrezas de traducción en áreas especializadas, así como la asimilación de una metodología práctica y funcional que permita al alumno optimizar las fases que configuran el proceso traslativo. 
Palabras clave: Manual virtual; Traducción especializada; Técnicas de traducción.

\section{INTRODUCCIÓN}

Con el fin de garantizar la capacitación del Graduado en Traducción e Interpretación por la Universidad de Córdoba para el ejercicio de las competencias que le son propias, en la Memoria Verifica de dicha titulación, se establece una serie de principios didácticos relativos a la adquisición de la competencia traductora general y especializada, así como el conocimiento de los fundamentos teóricos y metodológicos de la traductología, con sus aplicaciones prácticas.

Partiendo de esta base, este proyecto nace con un claro objetivo multidisciplinar, dado que no podemos obviar que en una sociedad basada en las tecnologías de la comunicación, debe ser nuestro cometido como docentes, no solo procurarle al estudiante unos conocimientos teórico-prácticos en traducción, sino capacitarlo para el acceso, búsqueda y selección de la información, utilizando herramientas y aplicaciones tecnológicas; así como fomentar en él destrezas que le preparen para el aprendizaje autónomo, como medio de desarrollo, innovación y responsabilidad profesional.

Según esto, este proyecto se orienta a la creación de material docente en soporte virtual para optimizar la práctica de la traducción especializada. En concreto, se ha diseñado un manual audiovisual, cuyo contenido se presenta de modo virtual a través de un conjunto de vídeos explicativos que reproducen, cada uno de ellos, los contenidos de los cuatro bloques disciplinares que lo configuran (traducción jurídico-económica, científico-técnica, humanístico-literaria y audiovisual). Este material audiovisual se va a alojar, junto a los textos, recursos bibliográficos, modelos de análisis, traducciones comentadas, etc., en la plataforma virtual Moodle (EXA ENOA2), de acceso abierto, para los alumnos de la Universidad de Córdoba.

Con este diseño virtual y responsivo, el estudiante puede acceder al material a cualquier hora, cualquier día de la semana y desde cualquier dispositivo móvil (teléfono, tablet, portátil). Del mismo modo, su estructura en itinerarios de especialización le permite, en un solo manual, tener a su dispocisión, una metodología práctica que reproduce de manera eficaz, y para varias disciplinas, todo el proceso de traducción: recepción del texto original, fase de comprensión, fase de documentación y fase de expresión.

Este material didáctico audiovisual favorece igualmente el aprendizaje por competencias, en particular determinadas competencias básicas y específicas que están establecidas en el plan de estudios de traducción, y que detallamos a continuación:

-Competencia Básica 5 (CB5). Desarrollo de la creatividad y capacidad de autoaprendizaje para emprender estudios posteriores con un alto grado de autonomía

-Competencia universidad 2 (CU2). Conocer y perfeccionar el nivel de usuario en el ámbito de las TIC.

-Competencia específica 1 (CE1). Analizar, crear y revisar profesionalmente todo tipo de textos y determinar valores y parámetros de variación lingüística y función textual.

-Competencia específica 8 (CE8). Ser capaz de analizar y sintetizar todo tipo de textos y discursos en las lenguas de trabajo: A, B y C.

-Competencia específica 11 (CE11). Capacidad de análisis morfosintáctico, semántico y discursivo de las lenguas de trabajo: B y C.

-Competencia específica 12 (CE12) Conocer las peculiaridades lingüísticas de las lenguas de trabajo y sus contrastes con respecto al idioma materno.

Consideramos pues que este material en soporte virtual de acceso abierto, que pretende configurarse como manual de autoaprendizaje de la traducción especializada, es una herramienta formativa esencial gracias a su accesibilidad, su estructura y diseño audiovisual; y se constituye, además, como complemento didáctico para el perfeccionamiento de las destrezas de traducción en áreas especializadas, así como la asimilación de una metodología práctica y funcional que permitirá al alumno optimizar las fases que configuran el proceso traslativo.

\section{OBJETIVOS}

o Desarrollo y perfeccionamiento de los conocimientos lingüísticos, culturales, temáticos y textuales, así como las destrezas necesarias para traducir.

o Adquirir la competencia traductora general y especializada.

o Conocer los principios teóricos y metodológicos de la traductología, con sus aplicaciones prácticas.

o Aprender de manera estratégica, autónoma y continua.

o Resolver problemas de traducción de textos especializados.

o Apoyar la enseñanza virtual y el empleo de herramientas virtuales.

o Fomentar el autoaprendizaje como medio de desarrollo, innovación y responsabilidad profesional a través de la formación práctica, continua y especializada.

o Disponer de destrezas documentales y desarrollar competencias profesionales en el uso de manuales y fuentes documentales generales y especializados. 
$\mathrm{O}$

Gestionar recursos de documentación para la resolución de problemas de traducción (competencia instrumental)

o Capacitar al estudiante para el acceso, búsqueda y selección de la información, utilizando las nuevas tecnologías de la información y comunicación.

o Movilizar conocimientos enciclopédicos, culturales y temáticos para resolver problemas de traducción (competencia extralingüística).

o Diferenciar las lenguas controlando las interferencias (competencia lingüística contrastiva)

o Practicar los procedimientos, estrategias y técnicas de traducción.

o Asimilar las convenciones pragmáticas y lingüísticas particulares de los géneros textuales.

o Aprender a analizar, sintetizar, razonar críticamente y tomar decisiones.

o Contribuir al desarrollo y mejora de la práctica traductológica a través del análisis textual y del conocimiento de las diversas estrategias metodológicas.

o Integrar las TIC como herramientas que contribuyan a que el estudiante abandone el papel de sujeto receptor y pase a ser un elemento activo de su aprendizaje.

o Asimilar y aprender a transmitir hábitos de actuación.

Para alcanzar los objetivos que acabamos de exponer contamos con el siguiente equipo de profesores que participan este proyecto docente:

\begin{tabular}{|l|l|}
\hline Juan Pedro Monferrer Sala & Beatriz Martínez Ojeda \\
Carmen Arnedo Villaescusa & Rafael Porlán Moreno \\
Azahara Veroz González & M del Mar Ogea Pozo $^{\text {Soledad Díaz Alarcón }}$ \\
Ángeles García Calderón & Lourdes Bonhome Pulido \\
\hline
\end{tabular}

\section{MATERIAL y MÉTODOS}

Para la creación de este manual didáctico, relacionamos a continuación los materiales que se han elaborado:

- Selección textual real, perteneciente a diferentes campos de especialización tales como el humanístico-literario, científico-técnico, jurídico-económico y audiovisual.

- Vídeo de la película Scoop, de Woody Allen.

- Plantilla de análisis textual del texto original y plantilla de análisis traductológico de la traducción al español.

- Se ha realizado una presentación power-point para cada texto-análisis que ilustra y aclara cada vídeo.

- Página web de la plataforma Moodle (EXA ENOA2)

Para la realización de los vídeos:

- videocámara

- micrófonos de solapa

- ordenador portátil y trípode

- equipo de grabación, edición y postproducción de UCODIGITAL
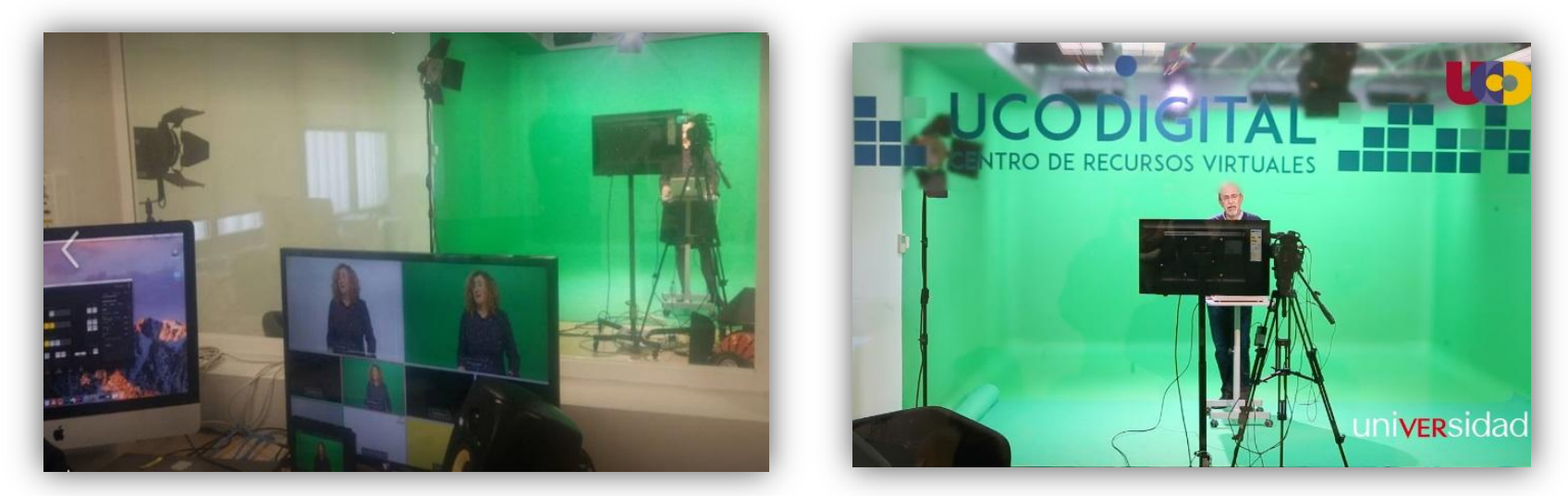

1. Instalaciones y equipos de UCODIGITAL (Campus de Rabanales)

\subsection{METODOLOGÍA}

La planificación, diseño y posterior implementación del soporte conceptual de este proyecto se asienta en la aplicación de los procedimientos que exponemos a continuación. 


\section{Fase preliminar:}

- Elección de los miembros que configuran el equipo de trabajo.

- Presentación, puesta en común y valoración crítica de la propuesta para este proyecto.

- Resolución de los contenidos sobre los que se asienta y planificación de su estructura.

- Selección y decisión de los soportes y recursos necesarios para su puesta en marcha.

- Reparto de tareas.

\section{Fase I:}

- Ordenación y distribución de tareas a los diferentes equipos de trabajo, en adelante Subproyectos:

o Subproyecto 1: Organización y coordinación de las fases del proceso y de los respectivos subproyectos.

o Subproyecto 2: Traducción jurídico-económico (Beatriz Martínez y Rafael Porlán)

o Subproyecto 3: Traducción científico-técnica. (Carmen Arnedo y Azahara Veroz)

o Subproyecto 4: Traducción humanístico-literaria. (Soledad Díaz y Angeles García)

o Subproyecto 5: Traducción audiovisual. (Mar Ogea)

o Subproyecto 6: Recursos y fuentes documentales. (Lourdes Bonhome y Fuensanta Guerrero)

o Subproyecto 7: Aula y soporte virtual (Juan Pedro Monferrer)

\section{Fase II:}

Subproyectos 2, 3, 4, 5 :

- Delimitación, determinación y selección del área de conocimiento especializada, temática y tipología textual.

- Desarrollo de objetivos, metodología específica y actividades concretas.

- Definición y diseño de modelo de análisis textual para el estudio de los textos originales explicados y comentados por los propios docentes, adaptados a los cuatro itinerarios y a las áreas de conocimiento, y que permita al alumno determinar, detectar y priorizar las especificidades de cada género textual y sus particularidades lingüísticas.

El modelo de análisis está estructurado en los siguientes apartados:

1. Introducción

2. Análisis discursivo del texto original.

2.1. Factores extratextuales

2.2. Factores intratextules

2.2.1. Nivel macrotextual

\subsubsection{Nivel microtextual}

\subsubsection{Nivel pragmático}

2.2.2.2. Nivel léxico-semántico

3. Conclusiones y objetivos de la traducción

4. Traducción al español del texto

5. Recursos bibliográficos

- Compilación del corpus textual que configurará los bloques temáticos (textos originales bilingües en francés y en inglés de la temática elegida).

Los textos seleccionados para este proyecto han sido los siguientes:

1. Análisis y traducción de texto científico-técnico (FR $>\mathrm{ES}$ )

CETISANDOZ 10 MG COMPRIMÉS PELLICULÉS

2. Análisis y traducción de texto científico-técnico (EN $>\mathrm{ES})$ BINOCULARS UNDER $100 \$$

3. Análisis y traducción de texto humanístico-literario (FR $>E S$ ) ILS SONT VOTRE ÉPOUVANTE ET VOUS ÊTES LEUR CRAINTE, de Thierry Jonquet

4. Análisis y traducción de texto humanístico-literario (EN $>\mathrm{ES})$ THE TESTAMENT, de John Grisham

5. Análisis y traducción de texto jurídico-económico $(\mathrm{FR}>\mathrm{ES})$ CONTRAT TYPE DE LOCATION OU DE COLOCATION DE LOGEMENT MEUBLÉ 


\section{Análisis y traducción de texto audiovisual (EN $>$ ES) SCOOP, de Woody Allen}

- Presentación de las propuestas de traducción:

a. Los docentes explican sus propuestas de traducción del corpus de textos, incidiendo en las características lingüísticas, tipologías textuales, convenciones pragmáticas, léxico especializado, etc.

b. Realización del análisis traductológico. Explicación y justificación del uso de los procedimientos, estrategias y técnicas de traducción más eficaces para cada caso, los retos que plantea cada disciplina y las fuentes documentales que ofrencen soluciones inequívocas.

- Elaboración de una bibliografía ${ }^{1}$ sustancial para cada área y temática, que abarca los ámbitos lingüísticos (en cada lengua), discursivos, traductológicos y pragmáticos.

\section{Subproyecto 6:}

- Proporciona y ayuda a los subproyectos 2, 3, 4, 5 en la búsqueda, selección y determinación de las herramientas, recursos y fuentes documentales generales y especializadas para cada actividad y materia.

\section{Fase III:}

- Presentación, puesta en común y valoración crítica del material elaborado y recursos didácticos.

- Diseño definitivo de los modelos de análisis textual y traductológico, y traducciones definitivas.

- Presentación de las referencias documentales seleccionadas.

- Preparación del guión que sustentará la grabación en vídeo.

Cada vídeo, de una duración de 12 minutos, se ha grabado en las instalaciones de UCODIGITAL en el campus de Rabanales. Cada profesor preparó una presentación powerpoint destacando los elementos textuales más relevantes, desde el punto de vista de la comprensión del texto original (en EN o FR) y su reexpresión en español, haciendo hincapié en las dificultades lingüísticas y pragmáticas de cada texto. Dicha presentación se ha apoyado en elementos icónicos y audiovisuales, de modo que la exposición resultase atractiva y fácilmente comprensible por el alumnado. He aquí algunos ejemplos:

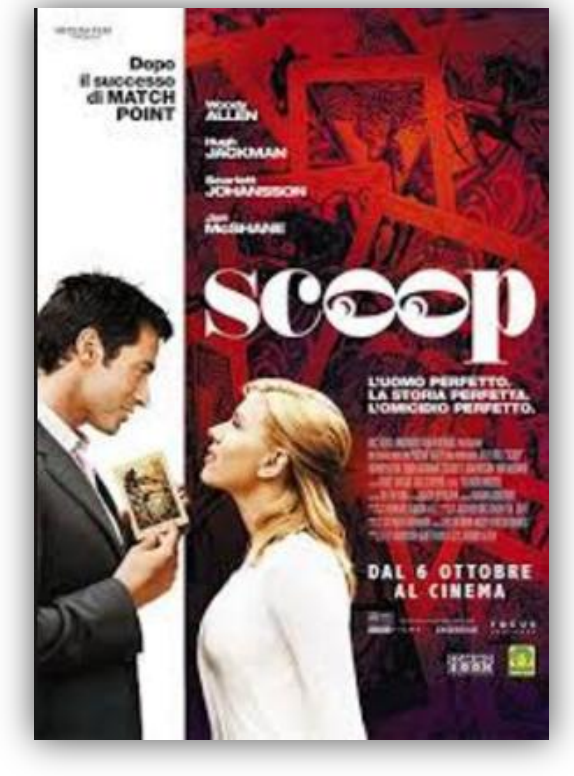

3. Traducción audiovisual $(\mathrm{EN}>\mathrm{ES})$

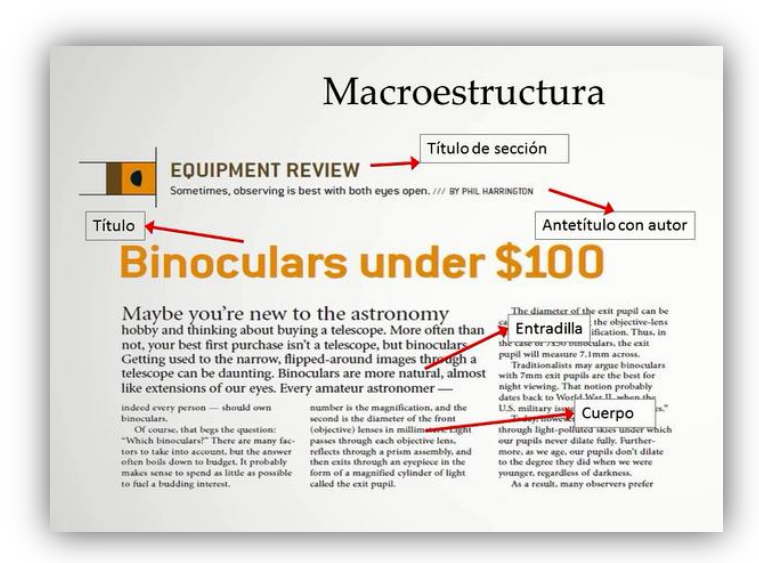

4. Traducción técnica (EN>ES)

\section{Fase IV:}

Subproyecto 7:

- Creación de la plataforma virtual en la que se ubicará el material.

\footnotetext{
${ }^{1}$ Una selección de los recursos bibliográficos trabajados en los diferentes análisis textuales que se han realizado en este proyecto se expone en el apartado \BIBLIOGRAFíA de la presente memoria.
} 
Hemos aprovechado el recurso del que dispone la Universidad de Córdoba, gracias a su Aula Virtual, de la plataforma Moodle (EXA ENOA2).

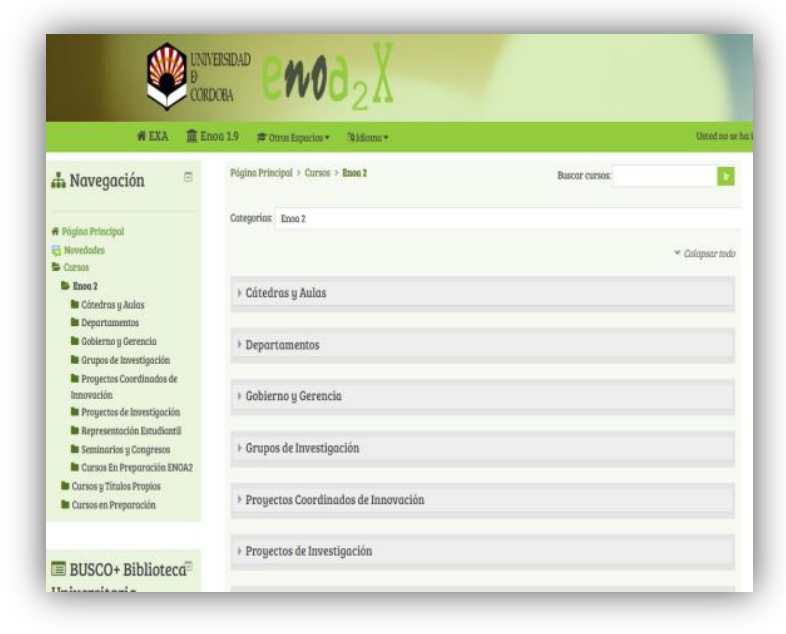

5. Plataforma EXA ENOA 2 (UCO)

- Disposición de los recursos necesarios (espacios y material) para la grabación de los vídeos.

- Coordinación y dirección de los agentes que grabarán el material audiovisual.

- Dirección de la grabación.

Fase V:

- Ordenación y disposición de todo el material en la plataforma.

- Promoción y difusión del recurso.

\section{RESULTADOS OBTENIDOS Y DISCUSIÓN}

Como hemos indicado en los objetivos planteados para este proyecto, los resultados obtenidos son los siguientes:

- Ofrecer un manual en soporte virtual, de acceso abierto, para la práctica de la traducción especializada.

- Perfeccionar las destrezas y técnicas de traducción en ámbitos especializados que ofrezcan soluciones reales para la resolución de los retos de traducción.

- Concienciar al alumno de la necesidad de una formación continua y autónoma.

- Fomentar una metodología TIC y audiovisual para la enseñanza-aprendizaje de la traducción especializada.

- Ofrecer un repositorio de recursos documentales esenciales y requeridos para la práctica profesional de la traducción especializada.

De este modo, se han realizado un total de 9 vídeos: una introducción, que justifica la creación del manual y 8 vídeos que se corresponden con la explicación de los textos elegidos por los respectivos docentes, analizados y traducidos al español. Textos que parten de las lenguas francesa e inglesa, así como un vídeo final que reúne un compendio bibliográfico de material didáctico de gran interés para el trabajo autónomo del alumno.

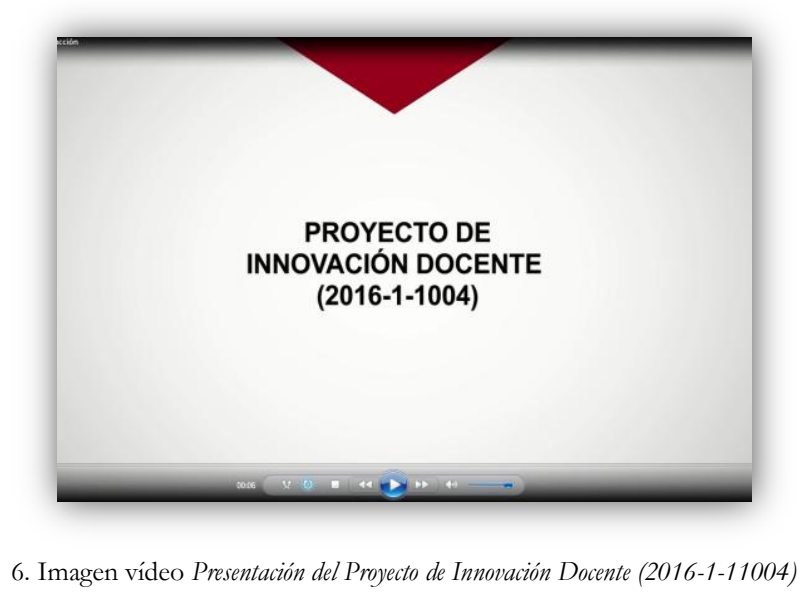



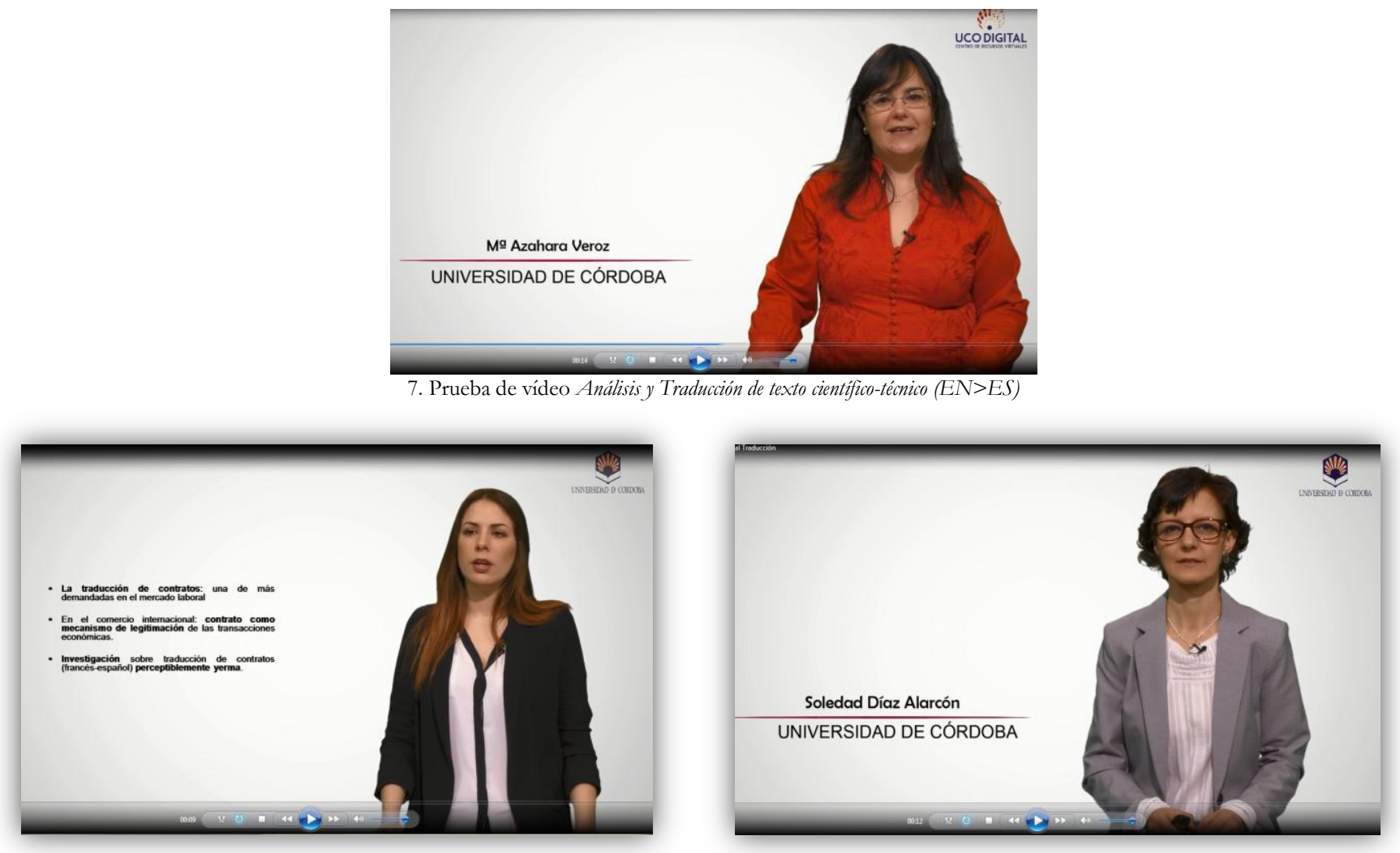

8. Imágenes de vídeos Análisis y Traducción de texto jurídico-económico (FR $>$ ES) y Análisis y Traducción de texto bumanístico-literario (FR $>$ ES)

En cuanto a los resultados que esperábamos al inicio del proyecto, mencionamos los siguientes logros:

- Mejora de los resultados académicos. A pesar de que hemos de reconocer que no tenemos los suficientes datos como para corroborar una mejora sustancial en el rendimiento académico de los alumnos, consideramos que desde un punto de vista formativo, el elemento audiovisual facilitará la comprensión y la adquisición de destrezas y habilidades en la práctica de la traducción especializada.

- Refuerzo de la motivación de los alumnos. El visionado de los vídeos fuera del aula les permite gestionar mejor su tiempo en función de su ritmo personal de aprendizaje, a lo que se une el hecho de que el material docente elaborado por los profesores está ideado en función del conocimiento de las carencias del alumnado, de ahí que se haya reforzado las competencias que aún no están adquiridas.

- Mayor implicación de los alumnos en el desarrollo de su aprendizaje. Las explicaciones en formato audiovisual favorecerán el aprendizaje autónomo.

- Satisfacción de los profesores con la metodología empleada. Los docentes que hemos participado en este proyecto estamos satisfechos con la forma en que se ha desarrollado el enfoque metodológico, dado que ha permitido un enfoque del proceso de enseñanza-aprendizaje, poniendo énfasis en el desarrollo competencial de los estudiantes. Así, ha sido posible que los alumnos ocupen el lugar central que se pretende desde el Espacio Europeo de Educación Superior.

- Implementación de nuevos recursos didácticos. Los docentes hemos tenido la oportunidad de conocer y explotar un recurso docente innovador y muy en consonancia con el desarrollo actual de las nuevas tecnologías como es la creación de material didáctico en formato virtual, lo que ha permitido conocer nuevas posibilidades didácticas fuera del recinto del aula.

\subsection{SUB EPÍGRAFE RESULTADOS OBTENIDOS Y DISCUSIÓN}

Este material didáctico se ha ubicado en la plataforma Moodle (EXA ENOA2) que el Aula Virtual de la Universidad de Córdoba pone a disposición de la comunidad educativa que la integra, por lo tanto tiene acceso abierto para todos los agentes implicados. La ubicación de todos los materiales didácticos (vídeos, artículos, textos, actividades, recursos documentales, etc.) en esta plataforma permite al alumno disponer en cualquier momento de un conjunto de recursos formativos esenciales para la práctica de la traducción especializada, seleccionados por especialistas en el materia. De este modo podrá consultarlos y trabajar con ellos directamente o descargarlos en su ordenador.

Su diseño responsivo (html 5) favorece además la visualización adecuada en cualquier dispositivo (móvil, tablet, portátil, etc.). Considerando que el alumnado de la Universidad de Córdoba, tanto de Grado como de Postgrado (másteres) está familiarizado con el uso de la plataforma Moodle, no tendrá dificultad alguna ni en el acceso, ni en la localización del material o la realización de las actividades. 
Asimismo, los miembros integrantes de este proyecto son docentes del Grado de Traducción e Intepretación, participan en el Máster (on line) de Traducción Especializada (inglés/francés/alemán-español) y forman parte de asociaciones nacionales de traducción, por lo que pueden promocionar y difundir este recurso a través de los medios que la Universidad de Córdoba pone a su disposición (plataformas, aula, boletín informativo de la facultad, redes profesionales, base de datos de alumnos y exalumnos del grado y del máster, etc.) y medios profesionales.

\section{CONCLUSIONES}

La preparación de este manual ha permitido a los docentes implicados en este proyecto una reflexión sobre la situación actual de la enseñanza-aprendizaje de la traducción especializada. En las diferentes sesiones y reuniones entre los docentes se han destacado las principales necesidades del alumnado de traducción así como las carencias y limitaciones de una formación exclusiva en el aula, en un horario y con unos recursos limitados.

Por estos motivos la creación de este manual virtual ha dejado patente que los recuros audiovisuales se configuran como herramienta imprescindible, no sólo para transmitir contenidos, sino por su enorme potencial educativo, lo que posibilita nuevos procesos de aprendizaje y de transmisión de conocimiento, a la vez que incorpora la interactividad como núcleo del proceso. Del mismo modo, valoramos la potencialidad del material multimedia como estrategia didáctica que alía aprendizaje y entretenimiento.

Estamos convencidos, por tanto, de que el manual virtual emanado de este proyecto docente facilitará al alumno de traducción el aprendizaje de destrezas y la adquisición de competencias necesarias para alcanzar el nivel experto en los diferentes campos de la traducción.

\section{AGRADECIMIENTOS}

Quisiera dar las gracias al equipo de profesionales que conforma UCODIGITAL, sin cuya ayuda, colaboración y apoyo no hubiese sido posible este proyecto. Agradecer igualmente a los compañeros que han participado en este trabajo, por su entusiasmo, esfuerzo y compromiso, gracias a todos ellos podemos ofrecer al alumnado de la UCO un material riguroso y eminentemente práctico para la adquisición de las compentencias que se le exigen a todo profesional de la traducción especializada.

\section{BIBLIOGRAFÍA}

Álvarez Calleja, A.“Crítica sistemática de la traducción”, Revista Alicantina de Estudios Ingleses 1993/6, p. 9-17.

Agost, R., "Los géneros de la traducción para el doblaje", en M. Duro (ed.), La traducción para el doblaje y la subtitulación, Cátedra, Madrid, 2001.

ASEDEF. "La redacción del prospecto. Recomendaciones para mejorar su comprensión", Documentos Asedef, 02, Asedef, Madrid. 2007.

BLANCAFORT, S. DE CAMVRA, S. y NAVARRO, MD. Funciones y utilidad del prospecto del medicamento. UAB, Barcelona, 2005.

Beltran, A. y CiverA, P. "Le contrat de bail dans le corpus de genres textuels pour la traduction GENTT", en Meunier, M.; Charret-Del Bove, M. Damette, E. La traduction juridique: Points de vue didactiques et linguistiques. Lyon, Publications du Centre d'Etudes Linguistiques, 2013, p. 281-323.

BORJA ALBI, A. "La traducción jurídica: aspectos textuales y didáctica", en Aproximaciones a la traducción, Madrid, Instituto Cervantes, 1999.

CAMPOS MARTíN, N . La traducción jurídica: los contratos. Estudio traductológico y terminológico comparado (francés/español), Granada, Comares, 2013.

CASINO, G.. "Sobre la legibilidad de la prosa de los medicamentos". 2005. [Consulta en línea: <http://www.essentialdrugs.org/efarmacos/archive/ 200511/msg00071.php> 06/02/2017]

Chaume Varela, F. Cine y traducción. Madrid, Cátedra, 2004.

CRUZ MartíneZ, M. S. y CruZ VARgas, M. L. "Características del inglés de los términos económico financieros: un enfoque traduccional / Characteristics of the english economic and financial terms: a translational approach". Entreculturas 2014/6.

DeLiSLE, J. L'analyse du discours comme méthode de traduction, Ottawa, Éditions de l'Université d'Ottawa, 1980.

DíAZ CinTAS, J. Y REMAEL, A.: Audiovisual translation: Subtitling. Manchester, St Jerome, 2007.

EuR-LEX. Access to European Union law. Multilingual display. [Consulta en línea: http://eurlex.europa.eu/homepage.html; 28/02/2007].

FuerTES-OlIVERA, P. A. y S. GÓMEZ-MARTíneZ. "Empirical assessment of some learning factors affecting Spanish students of Business English". English for Specific Purposes 2004/23.

GAMero PÉreZ, S. La traducción de textos técnicos. Descripción y análisis de textos (alemán-español), Barcelona, Ariel, 2001. 
Holl, I. "Técnicas para la traducción jurídica: revisión de diferentes propuestas, últimas tendencias", Hermeneus. Revista de Traducción e Interpretación, 2012/14, p. 191-216.

HURTADO AlBIR, A. Traducción y Traductología: Introducción a la traductología, Granada, Cátedra, 2004.

HuRTAdo AlBiR, A. "Métodos traductores y finalidad de la traducción. Propuesta de clasificación”, en Traducción y traductología. Introducción a la traductología, Madrid, Cátedra, 2004.

JONQUET, T. Ils sont votre épouvante et vous êtes leur crainte. Paris, Seuil, 2006

KOCH, P. y OESTERREICHER, W. Lengua hablada en la Romania: español, francés, italiano. Versión española Araceli López Serena, Madrid, Gredos, 2007, p.441

KRugman, P. R. El retorno de la economía de la depresión. Colección: Letras de Crítica, ed. Crítica, Barcelona, 2000.

LE FLOHIC, Alain. Réalité et fiction : interview de Thierry Jonquet. Lamballe, 2004.

López MARTíneZ, M.I. y Hernández SÁnCHEZ, E. "Los prospectos de los fármacos como paradigma de una comunicación inefectiva", Revista de Investigación Lingüistica, 2004/7: 147-160.

MACíAs OTÓN, E. "Los problemas conceptuales y socioculturales de la traducción jurídica (inglés/francés-español)", Revista de Llengua i Dret, n. 2015/63, p. 49-62.

MARTÍNEZ SIERRA, J. J. "De normas, tendencias y otras regularidades en traducción audiovisual", Estudios de Traducción, 2011/ 1, p. 151-170.

MUÑOZ, C. "Tipología textual y análisis para la traducción. Una tipología de géneros médicos”. En Translating science. Proceedings 2nd Internacional Conference on specialized Translation. 2002, p. 319-325.

Mirón CANELO, J. A.; AlONSO SARDÓN, M.; SÁENZ GONZÁLEZ, M.C. "Estimar la comprensión de los prospectos de los medicamentos". Atención Farmaceutica, 2000, vol. 2, no 4, p. 358-363.

NIDA, E. y TABER, Ch. La traducción: teoría y práctica. Madrid, Cristianidad. 1986.

PiZARRo SÁNCHEZ, I. (2010). “Análisis y traducción del texto económico, Inglés-Español”, Netbiblo, 2010.

RODRÍGUEZ ESPINOSA, M. "Subtitulado y doblaje como procesos de domesticación cultural”, en DURO, M. (Ed): La traducción para el doblaje y la subtitulación. Madrid, Cátedra, 2001.

TERMiUm Plus. Base de datos terminológica de la Oficina de Traducción del Gobierno de Canadá [Consulta en línea: http://www.btb.termiumplus.gc.ca/; 28/02/2007].

TOurY, G. Descriptive Translation Studies and Beyond, Amsterdam/ Philadelphia, John Benjamins, 1995,

UNIÓN EUROPEA. Inter-Active Terminology for Europe (LATE). [Consulta en línea: http://iate.europa.eu/; 28/02/2007].

ZABALBEASCOA TERRÁN, P. "La traducción del humor de Woody Allen o el arte de dominar la sutileza y la ironía", en Lorenzo, L.; Pereira, A. (eds.), Traducción subordinada (I) El doblaje, Universidade de Vigo, 2000. 